\title{
Hochprozentige Zahnaufhellungs-Präparate sind sicher
}

Nach Erscheinen der EU Kosmetik-Direktive im Jahr 2012 mit ihrer Begrenzung von kosmetischen Zahnaufhellungs-Produkten auf 6\% $\mathrm{H} 2 \mathrm{O} 2$ sind bis heute Stimmen laut geworden, die die Sicherheit höherprozentiger Peroxid-Präparate anzweifeln, obwohl diese als medizinische Präparate nur in der Zahnarztpraxis anzuwenden sind. In einem 1. Review, der im Herbst 2014 erschien, hatte sich die Autorin, Frau Prof. Olga Polydorou (Universität Freiburg), bereits mit der Zweckmäßigkeit und den Indikationen solcher Präparate befasst. Ihr Fazit: Behandlungen mit höherprozentigen Mitteln aus der Hand des Zahnarzts ermöglichen Ergebnisse, die auf anderen Wegen kaum oder nur mit deutlich höherem Aufwand erzielbar wären; zugleich ist Zahnaufhellung konservativer und weniger invasiv als alternative restaurative Maßnahmen. Der nun vorgestellte Review stellt die Sicherheit in den Mittelpunkt. Dazu wurde eine umfangrei- che Pubmed-Recherche durchgeführt; insgesamt wurden fast 300 Studien gesichtet und bewertet. Die Aussagekraft und der Vergleich der Ergebnisse waren nicht einfach, da sehr verschiedene Studien-Designs zu beachten waren. Außerdem wurden teilweise keine Fertig-Produkte, sondern Chemikalien (z. B. H2O2Lösungen) zum Test herangezogen; die Applikations-Arten und Zeiten waren ebenfalls zum Teil wenig praxisgerecht.

In der Schlussfolgerung fand Polydorou, dass sowohl Zahnaufhellungs-Präparate bis 6\% $\mathrm{H} 2 \mathrm{O} 2$ als auch mit höheren $\mathrm{H} 2 \mathrm{O} 2$ Anteilen sicher anzuwenden sind, ohne Langzeit-Schäden hervorzurufen. Voraussetzung dazu ist eine korrekte Handhabung, die jedoch gerade bei den höherprozentigen Präparaten durch die alleinige Anwendung in der zahnärztlichen Praxis besonders verlässlich gewährleistet ist. Sie kommt daher zu dem Schluss, dass bei-

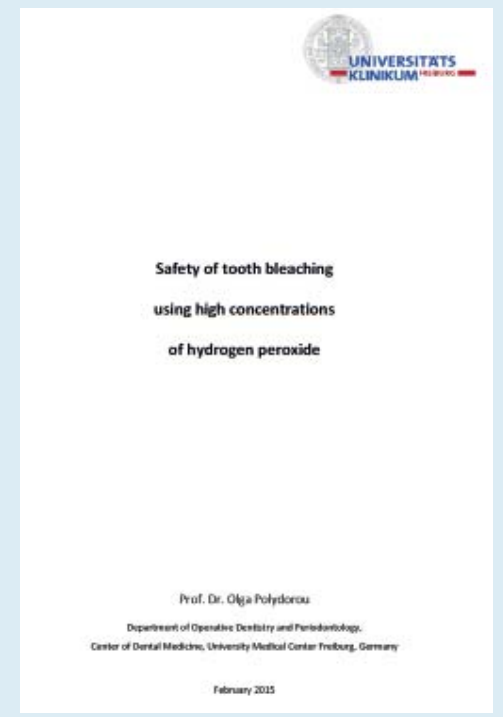

de Produktgruppen - kosmetische und medizinische - zur Verfügung stehen sollten, um je nach Patientenfall und Indikation eingesetzt werden zu können. Der Zahnarzt ist damit in der Lage, stets die effektivste und minimalinvasivste Behandlung auszuwählen.

Nach einer Pressemitteilung der Ultradent Products GmbH, Köln Internet: www.ultradent.com 